\title{
A Generic Energy-Conserving Discrete Element Modelling Strategy for Concave Particles Represented By Surface Triangular Meshes
}

\author{
Y. T. Feng* \\ Zienkiewicz Centre for Computational Engineering, College of Engineering, Swansea University, UK
}

\begin{abstract}
A generic energy-conserving linear normal contact model for concave particles in the discrete element method (DEM) is presented in the current paper. It is derived based on a recently enhanced general energy-conserving contact theory for arbitrarily shaped particles. A set of more effective evaluation schemes required in the model are also given, which shows that only the intersection boundary between two contact shapes, instead of their contact region or surfaces, is required to be explicitly obtained, thereby substantially improving both efficiency and applicability of the proposed contact model over the previous version. A surface triangular mesh is used to represent any $3 \mathrm{D}$ concave particle. The computational issues associated with the contact of two surface triangulated 3D shapes, including the contact detection, the determination of intersection boundary segments, the computation of contact features and parallelisation, critical time step, and friction and damping treatment for multiple contacts are described in detail. Two sets of numerical examples involving various concave 3D shapes with a large number of surface triangles are presented to demonstrate either the superb energy-conserving property of the proposed model model, or its effectiveness, robustness and universal nature for wider and more complex problems.
\end{abstract}

KEYWORDS: Concave shapes, Energy conservation, Linear normal contact model, Triangular mesh representation

\section{Introduction}

There has been a growing demand in the discrete element community to enhance the modelling capability of the discrete element method (DEM) [1 for real particles encountered in industrial applications, as particle shape has long been recognised as one of the most important factors that affect the physical behaviour of granular systems. Since the 1980s, continuous efforts have been made to extend the disc/sphere-based classic DEM to other shapes. The first set of shapes include single analytical function expressed geometric entities: ellipses [2, 3], ellipsoids [4, super-quadrics [5] and super-ellipsoids [6]. These shapes can be enriched by piecing together their surface patches to form more complex $3 \mathrm{D}$ shapes, such as poly-ellipsoids [7, 8] and poly-super-ellipsoids [9]. These shapes are, however, still characterised by their smooth surfaces (at the microscopic level) and convex nature, which are still far from real particle shapes.

Another set of shapes have edges and sharp corners in common and include cylinders [10, polygons [11] and polyhedra [12, 13, 14, 15]. The challenge associated with these shapes

*e-mail: y.feng@swansea.ac.uk 
is the normal discontinuity at edges and vertices that imposes difficulties on characterising their contact normal and overlap. One solution is to "round" the edges and/or vertices of these shapes using the dilation technique [16, 17, 18, 19], which is equivalent to applying the Minkowski sum operation to a base shape (line segment, polygon, polyhedron, or cylinder) and a (small) disc/sphere, where the radius of the disc/sphere controls the roundness of the resulting dilated or rounded smooth shape. Note that the Minkowski sum is a very powerful construction tool that can be used in a multi-level hierarchical fashion to generate much more complicated geometric shapes.

More recently, sophisticated basis functions have been utilised to enrich the representation of particle shapes. These include Fourier series for 2D shapes [20, NURBS (or B-spline) [21] or spherical harmonic [22, 23] for 3D objects, and iso-geometric for 3D surfaces [24, 25]. The distinct advantage of these shapes over those previously mentioned is that these shapes can be constructed either as convex or concave, and therefore are more representative of real particles. However, the best way to acquire realistic particle shapes is to employ various 3D shape digitalisation technologies such as laser and computed tomography (CT) scanning. The acquired 3D model is represented either as a discrete pixel/voxel cloud, a polygonal/triangular surface mesh or other forms. By applying some of those sophisticated basis functions such as spherical harmonics, a smooth representation of a 3D digital model can also be obtained (see [22, 23] for adopting such an approach). A more comprehensive review on particle shape representations can be found in [26].

In accompanying the development of various schemes to represent the shape or morphology of a non-disc/spherical particle, the corresponding (normal) contact models have also been developed in tandem. A complete normal contact model should determine the contact geometry of the two shapes concerned and relates these geometric features, if the contact exists, to the contact forces to be applied to the contacting particles. In this sense, the (local) contact detection, which aims to establish if two particles are in contact, is included in the geometric aspect of a contact model. The contact geometric features associated with a contact include the contact region/surfaces, contact volume/area/overlap, contact normal direction, and contact point. A normal contact model should unambiguously define the direction, contact point and magnitude of the normal contact force for any possible configuration of the two shapes concerned. In this work, the normal direction, contact point and magnitude are termed the contact features of a normal contact force.

Unlike discs/spheres where their contact geometries are well defined, this is no longer the case for irregular particles and various definitions may exist for a particular geometric feature. For instance, for a corner-corner contact between two polygons/polyhedra [11, 12, 14], defining the contact normal direction may become an issue due to boundary normal discontinuities of polygons/polyhedra at their edges/vertices. Equally, defining the overlap and the contact point in this particular contact situation also becomes an issue.

A more serious problem for irregularly shaped particles in general is that the normal contact features for a particular shape are defined in a non-consistent and "ad-hoc" fashion. Moreover, the definition of the magnitude of a normal contact force is typically independent of how the contact geometric features are defined. In the other words, the geometric and physical aspects of a normal contact model are essentially decoupled. Both the inconsistency between the definition of the contact geometric features and, to a lesser extent, the decoupling between the contact geometry and the contact force can cause numerical instability in DEM simulations which can often be difficult to be rectified.

The above discussion highlights the need to develop a more fundamental contact theory that can provide a generic framework so that the contact of arbitrarily shaped particles can be 


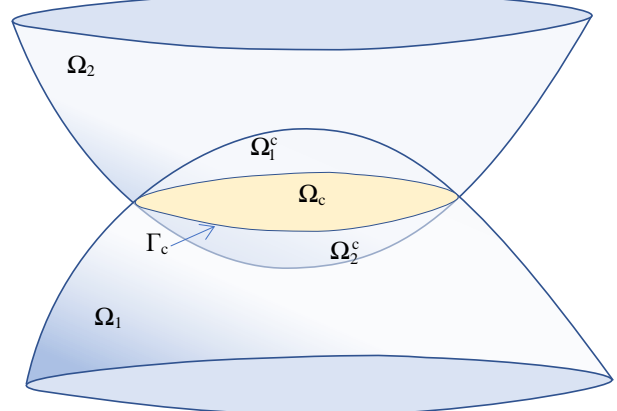

(a)

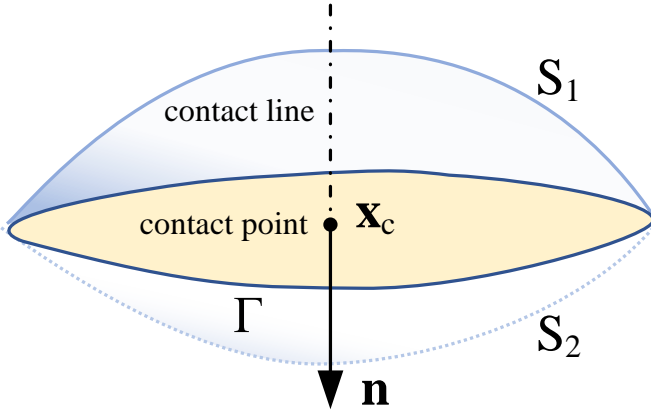

(b)

Figure 1: Two arbitrarily shaped bodies $\Omega_{1}$ and $\Omega_{2}$ in contact: (a) the contact geometry; (b) the contact features

modelled in a more uniform way. An attempt to make progress towards this goal was started in [11] where a contact energy function of the contact area is introduced to characterise the contact energy state between two polygons. The resulting normal contact model defines all the contact force features in a consistent manner and more importantly exhibits energy conservation. The model can also be extended to arbitrary $2 \mathrm{D}$ shapes as any $2 \mathrm{D}$ shape can be discretised into a (convex or concave) polygon. The same concept is applied to polyhedra and arbitrary 3D shapes in [13, 14], and the underlying contact theory is also highlighted in [14, 27]. This energy-conserving contact theory is further enhanced and generalised in a recent work [28]. In another closely related work [29], new numerical procedures are developed which can significantly improve the computational performance of the normal contact model over the original version [14] by avoiding the explicit construction of the contact surfaces of two contacting particles.

The current paper applies this contact volume based normal contact model to 3D concave shapes represented by surface triangular meshes. The contact model will be presented in the next section, together with the description of the new computational procedures developed in [29]. Several numerical aspects of using this contact model for two surface triangulated concave shapes in contact, including the contact detection, the computation of the contact features and parallelisation, critical time step, and friction and damping treatment for multiple contacts are discussed in Section 3. Two sets of examples involving various concave shaped 3D particles are provided in Section 4 to further demonstrate the superb energy-conserving property of the contact model and its robustness and universal nature for wider and more complex applications.

\section{Contact Volume Based Energy-Conserving Normal Contact Model for 3D Shapes}

\subsection{The Linear Normal Contact Model}

Consider a general contact between two arbitrary 3D shapes $\Omega_{1}$ and $\Omega_{2}$, as shown in Figure 1(a). Let $\Omega_{c}$ be the contact region, and $S_{1}$ and $S_{2}$ be the contact surfaces belonging to $\Omega_{1}$ and $\Omega_{2}$ respectively. Define $V_{c}$ to be the volume of the contact region $\Omega_{c}$. Following the theoretical development in [29] and choosing the contact energy function $w$ to be a linear function of $V_{c}$ will lead to the following linear normal contact model which fully determines 
all the contact force features.

[Contact volume based linear normal contact model]: Let the contact energy $w=k_{n} V_{c}$ be a linear function of the contact volume $V_{c}$, where $k_{n}$ can be viewed as a 'spring' stiffness. The normal force $\mathbf{F}_{n}$ exerted on Body 1 can be obtained as

$$
\mathbf{F}_{n}=-\nabla_{x} w\left(V_{c}\right)=-k_{n} \mathbf{S}_{n}
$$

where $\mathbf{S}_{n}$ is the vector area of the contact surface $S_{1}$

$$
\mathbf{S}_{n}=\int_{S_{1}} d \mathbf{S}
$$

with the projected or scalar contact area $S_{n}=\left|\mathbf{S}_{n}\right|$. Then

1) The unit normal contact vector $\mathbf{n}$ is defined as the negative unit direction of $\mathbf{S}_{n}$

$$
\mathbf{n}=-\mathbf{S}_{n} / S_{n}
$$

2) The magnitude $F_{n}$ is identified as

$$
F_{n}=k_{n} S_{n}
$$

3) The contact point $c$ is determined by the coordinates $\mathbf{x}_{c}$

$$
\mathbf{x}_{c}=\frac{\mathbf{n} \times \mathbf{G}_{n}}{S_{n}}+\lambda \mathbf{n}, \text { with } \mathbf{G}_{n}=\int_{S_{1}} \mathbf{x} \times d \mathbf{S}
$$

where $\lambda$ is a free parameter, and its all possible values define the normal contact line.

The normal force $\mathbf{F}_{n}^{\prime}$ exerted on Body 2 by Body 1 is

$$
\mathbf{F}_{n}^{\prime}=-\mathbf{F}_{n}=-F_{n} \mathbf{n}
$$

and can be applied to the same contact point (but in Body 2) or any point on the contact line.

All the derivations that lead to this energy-conserving linear normal contact model can be found in [28, 29]. The main contact geometric features defined by the model are shown in Figure 1(b). Note that although the contact force features are obtained from the view point of Body 1 (or the contact surface $S_{1}$ to be precise), due to symmetry, these features and all the formulae presented blow can be equally obtained in terms of Body 2 or the contact surface $S_{2}$.

This linear normal contact model has the following distinct properties [29]: 1) energy conservation guaranteed for any elastic impact; 2) applicable to any shape, $2 \mathrm{D}$ or $3 \mathrm{D}$, convex or concave; 3) capable of automatically handling both single or multiple contact scenarios; and 4) highly parallelizable. The related computational issues are discussed below. 


\subsection{Computational Issues}

From a computational point of view, in order to fully determine the features of the normal contact force, the key steps of the above contact model involves evaluating the following two integrals over the contact surface $S_{1}$ (or $S_{2}$ ):

$$
\mathbf{S}_{n}=\int_{S_{1}} d \mathbf{S} ; \quad \mathbf{G}_{n}=\int_{S_{1}} \mathbf{x} \times d \mathbf{S}
$$

where $\mathbf{S}_{n}$ determines the force or normal direction $\mathbf{n}$ and the force magnitude $F_{n}$; while $\mathbf{G}_{n}$ is essential to determine the contact point $\mathbf{x}_{c}$ or the contact line. The two integrals indicate that the contact surface $S_{1}$ (and equally $S_{2}$ ) solely determines the contact force features, while the contact volume $V_{c}$ is not present. Thus the whole contact region $\Omega_{c}$ does not need to be explicitly constructed. However, the contact surface $S_{1}$ or $S_{2}$ may have to be obtained.

For the contact of two convex polyhedra, there are several techniques [14, 29, 31, 32] that can be used to compute $\mathbf{S}_{n}$. Nevertheless, for other non-spherical shapes, the explicit construction of $S_{1}$ (or $S_{2}$ ) seems inevitable. This is clearly a computationally intensive step, which makes the currently contact model computationally expensive and less appealing than other existing contact models for non-spherical particles, despite the fact that the contact model offers superior numerical accuracy and stability and it is also applicable to both convex and concave shapes.

The above computational issue has now been largely resolved in [29] by the development of alternative schemes to evaluate $\mathbf{S}_{n}$ and $\mathbf{G}_{n}$, in which the integral domain of the two integrals is changed to the intersection boundary $\Gamma$ between the two contact surfaces $S_{1}$ and $S_{2}$, so that neither of the surfaces needs to be explicitly obtained. As a result, the computational costs associated with evaluating $\mathbf{S}_{n}$ and $\mathbf{G}_{n}$ (and the contact force features) have been significantly reduced. These new computational schemes are summarised below. The details can be found in [29].

\subsubsection{New computational schemes for $\mathrm{S}_{n}$}

Consider a (open) contact surface $S_{1}$ and its boundary $\Gamma$ which is the intersection between $S_{1}$ and $S_{2}$. Let $\Gamma$ be properly oriented so that its positive (tangential) direction is consistent with the outer normal of the surface as shown in Figure 2. The correction orientation of $\Gamma$ can be attained if its positive direction $\tau$ at an intersection point is determined by:

$$
\boldsymbol{\tau}=\mathbf{n}_{1} \times \mathbf{n}_{2}
$$

where $\mathbf{n}_{1}$ and $\mathbf{n}_{2}$ are the outer normals of the two surfaces $S_{1}$ and $S_{2}$ at the intersection point respectively. Below, the boldface $\boldsymbol{\Gamma}$ is used to denote the properly oriented $\Gamma$.

Now an equivalent but more general expression for $\mathbf{S}_{n}$ can be stated as

$$
\mathbf{S}_{n}=\int_{\bar{S}} d \mathbf{S} \quad \forall \bar{S}: \partial \bar{S}=\boldsymbol{\Gamma}
$$

where $\bar{S}$ can be any surface that has the same oriented boundary $\boldsymbol{\Gamma}$ as $S_{1}$. This expression may lead to a more effective evaluation if an ideal replacement surface $\bar{S}$ can be chosen in a way that it can offer the best computational efficiency in evaluating the integral.

One such candidate is shown in Figure 2 which is a conical surface with the origin as its apex and $\Gamma$ as its base. As this special surface is entirely determined by $\Gamma$, this further implies that it is the intersection curve $\Gamma$ of the two contacting bodies that dictates $\mathbf{S}_{n}$ or the normal 


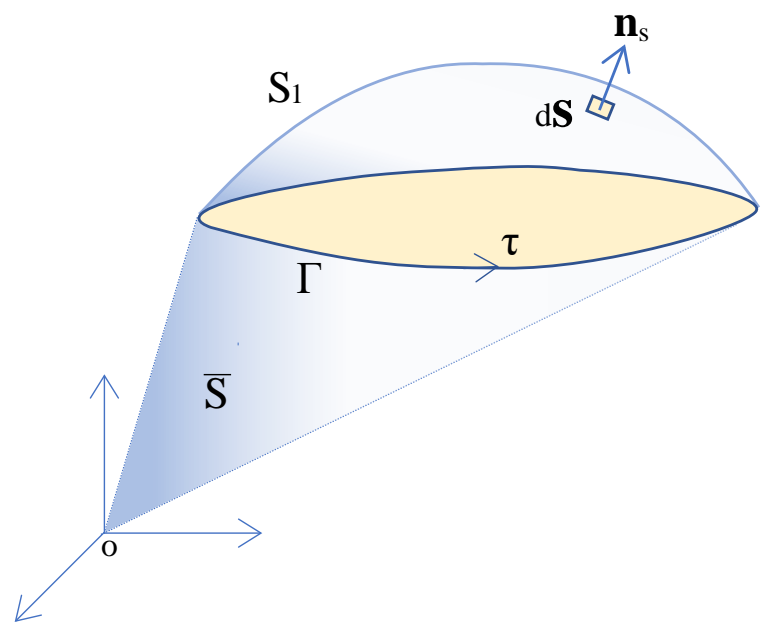

Figure 2: The contact surface $S_{1}$ with the boundary $\Gamma$ and one 'conical' replacement surface $\bar{S}$

direction, rather than the contact region $\Omega_{c}$ or surfaces $S_{1}$ and $S_{2}$. This assertion is fully supported by another equivalent expression for $\mathbf{S}_{n}$ :

$$
\mathbf{S}_{n}=\frac{1}{2} \oint_{\Gamma} \mathbf{x} \times d \boldsymbol{\Gamma}
$$

This expression is significant in that: 1) it mathematically proves that $\mathbf{S}_{n}$ is solely determined by the intersection $\Gamma$ of the two contacting bodies; and 2) it provides an alternative and more efficient procedure to evaluate $\mathbf{S}_{n}$ : the original surface integral over the contact surface $S_{1}$ (or $S_{2}$ ) is now replaced by a line integral over the intersection $\Gamma$.

Both (9) and (10) offer two alternative evaluation schemes for $\mathbf{S}_{n}$. When the conical surface $\bar{S}$ shown in Figure 2 is adopted as the replacement surface, the two schemes are identical. These two schemes will be used in Section 3 for two contacting particles that are triangulated and their intersection boundary $\Gamma$ becomes a discretised polyline.

\subsubsection{New computational schemes for $\mathbf{G}_{n}$}

Applying a similar argument to $\mathbf{G}_{n}$, an alternative integral for $\mathbf{G}_{n}$ can be written as

$$
\mathbf{G}_{n}=\int_{\bar{S}} \mathbf{x} \times d \mathbf{S}
$$

where $\bar{S}$ has the same meaning as in (9). Again, the special conical surface $\bar{S}$ used for computing $\mathbf{S}_{n}$ is an ideal option. The further derivation [29] also leads to another equivalent integral for $\mathbf{G}_{n}$ but over the intersection $\Gamma$ instead

$$
\mathbf{G}_{n}=\int_{S_{1}} \mathbf{x} \times d \mathbf{S}=-\frac{1}{3} \oint_{\Gamma} \mathbf{x} \cdot \mathbf{x} d \mathbf{\Gamma}
$$

Moreover, as $\Gamma$ is a boundary curve, it can be parameterised in principle by a scalar $t$. The above line integral can be further reduced to a definitive integral over an interval

$$
\mathbf{G}_{n}=-\frac{1}{3} \int_{t_{0}}^{t_{1}} \mathbf{x}(t) \cdot \mathbf{x}(t) d t
$$

where $\mathbf{x}\left(t_{0}\right)=\mathbf{x}\left(t_{1}\right)$ are the start and end points of the boundary $\Gamma$. 
Equation (11) uses a simple replacement surface $\bar{S}$ (which only needs to know the contact intersection $\boldsymbol{\Gamma}$ ) to evaluate $\mathbf{G}_{n}$. Equation (12) reduces this evaluation from a surface integral over $\bar{S}$ to a line integral over $\Gamma$, while Equation 13 further reduces it down to an integral over a parameter interval.

In summary, in the newly developed alternative schemes for $\mathbf{S}_{n}$ and $\mathbf{G}_{n}$, only the contact intersection boundary curve $\Gamma$ between the two shapes needs to be explicitly constructed, while the contact surface $S_{1}$ (or $S_{2}$ ) is no longer needed. Thus, these developments have significantly improved the computational efficiency over the original version [14] where $S_{1}$ has to be explicitly obtained. These new evaluation schemes for $\mathbf{S}_{n}$ and $\mathbf{G}_{n}$ also reduce the associated computational cost to an insignificant level in comparison with explicitly finding the intersection $\Gamma$.

Consequently, applying the proposed linear contact model to arbitrarily shaped particles (both convex and concave included), the main computational issue becomes the construction of the intersection boundary $\Gamma$ between the two shapes concerned. This is in general still not an easy task to fulfil for shapes that are described by (nonlinear) analytical functions, such as super-quadrics or spherical harmonics. However, when these shapes are represented by surface triangular meshes, obtaining the intersection boundary between two such shapes is reduced to finding the intersection lines between triangles of the two meshes. The detailed numerical implementation of the proposed linear contact model for surface triangulated shapes is discussed in the next section.

\subsection{Contact point/line}

After $\mathbf{G}_{n}$ is evaluated, the contact point $\mathbf{x}_{c}$ can be determined by (5). Because $\lambda$ is a free parameter, and can take any value in principle, this means that the contact point is not unique. When $\lambda$ takes different values, the point forms a line which is called the contact line. This non-uniqueness does not have any effect on the normal contact, but the location of the point on the contact line will have an impact on the tangential force. To resolve this issue for the tangential contact, an additional condition needs to be imposed. For instance, by introducing the concept of a minimal contact surface, a simple formula has been derived in [29] to determine the unique contact point. Alternatively, the two intersection points of the contact line with the two contact surfaces can be obtained, in particular when the surfaces are represented by triangular meshes as described in the next section. Then either one, or their middle point can be chosen as the contact point to ensure that the contact point does not locate outside the contact region. Although different choices of $\mathbf{x}_{c}$ can lead to different results, the difference is expected be be small.

\subsection{Single and Multiple contacts}

The preceding discussion is based on the assumption that only one set of $\mathbf{S}_{n}$ and $\mathbf{G}_{n}$ exists for each contact pair, and therefore a single contact situation is assumed. For concave contacts, however, multiple independent sets of $\mathbf{S}_{n}$ (and $\mathbf{G}_{n}$ ) may exist, each corresponding to one independent or single contact. As each single contact can be handled by the proposed model, multiple contact scenarios can be dealt with automatically. The detailed discussion of the related issues, including the classification of distinct and associated multiple contacts, can be found in 28 . The issues related to friction and damping are briefly addressed in Section 3.7 . 


\section{Surface Triangulated Non-spherical Particle Contact Model}

\subsection{Triangular Surface Mesh Represented 3D Shapes}

Surface triangulation is a universally applicable technique to represent any 3D shape, and has been widely used as one of the standard schemes for digitally representing geometric entities, including scanned objects. Triangulation can also be applied to a 3D shape that is analytically described by a mathematical function. For instance, a particular superquadric [33] that is analytically expressed in the $x, y$ and $z$ coordinates

$$
\left(\left|\frac{x}{a}\right|^{n_{2}}+\left|\frac{y}{b}\right|^{n_{2}}\right)^{n_{1} / n_{2}}+\left|\frac{z}{c}\right|^{n_{1}}-1=0
$$

can be parametrised by the following form (in the first quadrant)

$$
x=a \cos ^{2 / n_{2}}(\alpha) \sin ^{2 / n_{1}}(\theta) ; y=b \sin ^{2 / n_{2}}(\alpha) \sin ^{2 / n_{1}}(\theta) ; z=c \cos ^{2 / n_{1}}(\theta)
$$

in the parameter space $(\alpha, \theta) \in[-\pi, \pi] \times[0, \pi]$. By specifying a certain level of resolution, the 2D parameter space $(\alpha, \theta)$ can be triangulated. Next mapping back the triangulation to the $x, y$ and $z$ coordinates using (15) will result in a surface triangulation of the superquadric. A similar triangulation procedure is used to represent star-shaped 3D particles in 22, 23. A recent significant improvement on the triangulation of spherical harmonic particles can be found in 30 .

In practice, there are many $\mathrm{CAD}$ toolkits that can provide a triangular surface mesh for a 3D shape. In most DEM simulations of industrial problems, wall boundaries can be very complex, and are typically defined by CAD models and imported into DEM simulations as surface triangulated geometric shapes. Thus, the procedures presented below are equally applicable to a particle-wall contact scenario, where both the particle and the wall can be any shape - convex or concave. Note that a reasonable discretisation level of a 3D particle often produces a large number of surface triangles. The following subsections discuss the main computational issues associated with applying the proposed contact model to two surface triangulated shapes.

\subsection{Construction of Intersection Line Segments}

For two shapes whose surfaces are discretised into triangular meshes, their intersection boundary becomes a closed polyline $\Gamma_{h}$, which is a discretised version of $\Gamma$, as shown in Figure 3 . For multiple contacts, $\Gamma_{h}$ consists of multiple independent and closed polylines.

The linear segments of $\Gamma_{h}$ are formed by intersecting all the individual triangles from the two surfaces. The basic numerical procedure involves determining the line segment of the intersection (if exists) between two given triangles from each surface, and this can be done in a straightforward manner. There are two intersection points if two triangles are in contact. However, in the special case when two triangles lie on the same plane, it is regarded as no contact. The start and end points of the segment should be properly ordered to ensure that the orientation of the segment is consistent with the orientation of $\Gamma$ defined by (8). The positive direction $\tau$ of the segment is given by

$$
\boldsymbol{\tau}=\mathbf{n}_{1} \times \mathbf{n}_{2}
$$

where $\mathbf{n}_{1}$ and $\mathbf{n}_{2}$ are respectively the outer normal directions of the two triangles concerned. 


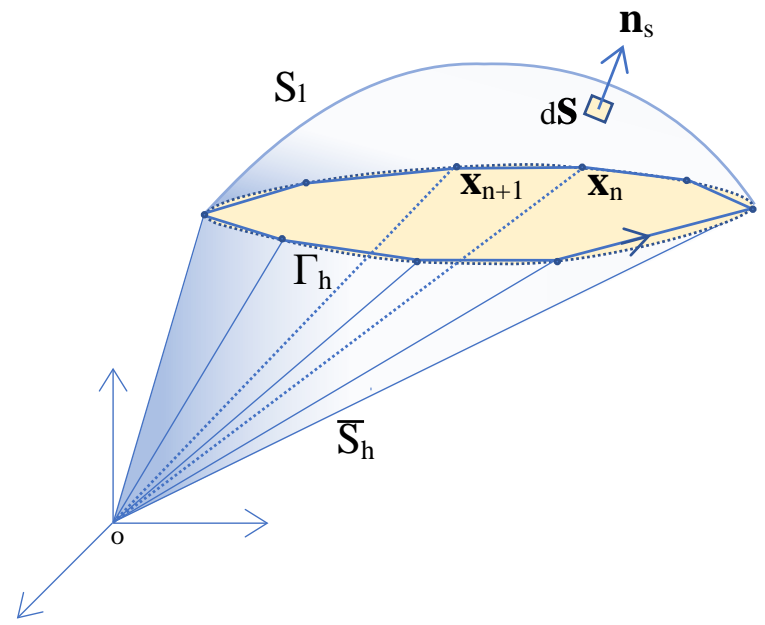

Figure 3: The contact surface $S_{1}$ with the discretised boundary polyline $\Gamma_{h}$ and also the discretised replacement surface $\bar{S}_{h}$

In order to compute $\mathbf{S}_{n}$ and $\mathbf{G}_{n}$ based on all the intersection segments determined, each line segment can be locally parameterised. Denote the $i$-th line segment as $\Delta \Gamma_{i}$ with the start and end points being $\mathbf{x}_{i}$ and $\mathbf{x}_{i+1}$, and let $\Delta \mathbf{x}_{i}=\mathbf{x}_{i+1}-\mathbf{x}_{i}$. Introduce a local linear coordinate system on the segment with parameter $t \in[0,1]$, the parametric equation and the infinitesimal increment of the segment are

$$
\mathbf{x}(t)=\mathbf{x}_{i}+t \Delta \mathbf{x}_{i} ; \quad d \boldsymbol{\Gamma}=\Delta \mathbf{x}_{i} d t
$$

\subsection{Computation of $\mathrm{S}_{n}$}

Section 2.2.1 presents two alternative schemes to evaluate $\mathbf{S}_{n}$ as below

$$
\mathbf{S}_{n}=\int_{\bar{S}} d \mathbf{S}=\frac{1}{2} \oint_{\Gamma} \mathbf{x} \times d \mathbf{\Gamma}
$$

As $\Gamma$ has been discretised into $\Gamma_{h}$, the corresponding discretised version of the second scheme is

$$
\mathbf{S}_{n}=\sum_{i=1}^{m} \mathbf{S}_{n}^{i}=\frac{1}{2} \sum_{i=1}^{m} \int_{\Delta \Gamma_{i}} \mathbf{x} \times d \boldsymbol{\Gamma}
$$

where $m$ is the total number of segments of $\Gamma_{h}$; and $\mathbf{S}_{n}^{i}$ is the contribution from the $i$-th segment

$$
\mathbf{S}_{n}^{i}=\frac{1}{2} \int_{\Delta \Gamma_{i}} \mathbf{x} \times d \boldsymbol{\Gamma}=\frac{1}{2}\left[\int_{0}^{1} \mathbf{x}(t) d t\right] \times \Delta \mathbf{x}_{i}=\frac{1}{2} \mathbf{x}_{i} \times \mathbf{x}_{i+1}
$$

which is geometrically equivalent to the signed area of the triangle formed by the origin $\mathbf{o}$ and the two end points $\mathbf{x}_{i}$ and $\mathbf{x}_{i+1}$. Then the discretised version to evaluate $\mathbf{S}_{n}$ reduces to a simple formula

$$
\mathbf{S}_{n}=\frac{1}{2} \sum_{i=1}^{m} \mathbf{x}_{i} \times \mathbf{x}_{i+1}
$$

Note that the triangles formed by the origin and the end points of all the segments in $\Gamma_{h}$ form a triangulated mesh of the conical replacement surface $\bar{S}$, which is denoted as $\bar{S}_{h}$ as shown in Figure 3. It is easy to verify that $\mathbf{S}_{n}^{i}$ is the same as the signed area of the $i$-th triangle. Consequently the formula (21) is identical to use $\bar{S}_{h}$ as the replacement surface in the first scheme of (18). 


\subsection{Computation of $\mathrm{G}_{n}$}

Section 2.2.1 presents three alternative schemes (11) and (12) to evaluate $\mathbf{G}_{n}$

$$
\mathbf{G}_{n}=\int_{\bar{S}} \mathbf{x} \times d \mathbf{S}=-\frac{1}{3} \oint_{\Gamma} \mathbf{x} \cdot \mathbf{x} d \mathbf{\Gamma}=-\frac{1}{3} \int_{t_{0}}^{t_{1}} \mathbf{x}(t) \cdot \mathbf{x}(t) d t
$$

When $\bar{S}$ is replaced by $\bar{S}_{h}$ and $\Gamma$ by $\Gamma_{h}$, their corresponding discretised versions are summarised below. The detailed derivation can be found in [29].

For the first scheme, let $T_{i}$ be the $i$-th triangle with $\mathbf{n}_{i}$ as its unit outward normal and $\mathbf{c}_{i}=\left(\mathbf{o}+\mathbf{x}_{i}+\mathbf{x}_{i+1}\right) / 3$ (where $\mathbf{o}$ is the origin) be the centroid. The integral of $\mathbf{G}_{n}$ over $T_{i}, \mathbf{G}_{n}^{i}$, can be explicitly expressed as

$$
\mathbf{G}_{n}^{i}=\int_{T_{i}} \mathbf{x} \times d \mathbf{S}=\int_{T_{i}} \mathbf{x} \times \mathbf{n}_{i} d A=\mathbf{c}_{i} \times \mathbf{S}_{n}^{i}
$$

Then the total integral $\mathbf{G}_{n}$ can be computed as

$$
\mathbf{G}_{n}=\sum_{i=1}^{m} \mathbf{c}_{i} \times \mathbf{S}_{n}^{i}
$$

For the second and third schemes, the contribution from the $i$-th segment $\Delta \Gamma_{i}$ can be derived as

$$
\int_{\Delta \Gamma_{i}} \mathbf{x} \cdot \mathbf{x} d \boldsymbol{\Gamma}=\left[\int_{0}^{1}\left(x^{2}+y^{2}+z^{2}\right) d t\right] \Delta \mathbf{x}_{i}=\mathbf{x}_{i} \cdot \mathbf{x}_{i+1}+\frac{1}{3} \Delta \mathbf{x}_{i} \cdot \Delta \mathbf{x}_{i}
$$

which gives the discretised total $\mathbf{G}_{n}$ as

$$
\mathbf{G}_{n}=\sum_{i=1}^{m} \mathbf{G}_{n}^{i}=-\frac{1}{3} \sum_{i=1}^{m}\left(\mathbf{x}_{i} \cdot \mathbf{x}_{i+1}+\frac{1}{3} \Delta \mathbf{x}_{i} \cdot \Delta \mathbf{x}_{i}\right) \Delta \mathbf{x}_{i}
$$

It can be verified that both (23) and (25) are identical, but computationally, the latter may be slightly more effective.

\subsection{Contact Detection}

In principle, the procedure presented in Section 3.2 can also be treated as a contact detection procedure for two triangulated shapes: when no intersection segment is found between two triangular meshes, the two shapes are not in contact. However, to check all possible pair-wise combinations between two sets of triangles to obtain the intersection segments can be very computationally intensive, particularly for shapes with a large number of triangles. Thus the computational cost involved can dictate the overall performance of a DEM simulation that employs the proposed contact model for particles with many surface triangles.

The key to improving the efficiency of the triangle intersection determination is to effectively exclude pairs of triangles that cannot be in contact from the intersection computation. There are slightly different approaches to achieve this goal, but all involve the combination with the so-called global search of the DEM procedure, as outlined in [34. The global search stage typically uses axis aligned bounding boxes to represent particles, excludes box pairs that are not in contact, and produces a potential particle contact list in the system.

Approach 1: Using one bounding box for a whole particle in the global search. In this approach, the number of bounding boxes in the global search is equal to the number of particles, resulting in a minimal cost in the global search. Then in the particle-particle 
contact detection stage, another contact search is conducted but only locally for the two particles (or two meshes) involved, where each triangle is represented by a bounding box. This local search should effectively exclude most triangle pairs that do not intersect. The actual intersection computation is only performed for the remaining triangle pairs. In this approach, the local search, which must be performed for all potentially contact particle pairs generated from the global search, can be the most time consuming part.

Approach 2. Using one bounding box for every surface triangle of a particle in the global search. In this approach the total number of bounding boxes involved in the global search can be substantial for large scale problems, resulting in a very high computational cost at this stage. However, in the particle-particle contact stage, no local search is required, and only triangle pairs that are identified as potential contact pairs will be involved in the intersection computation. Thus in this approach, a small portion of computational cost is associated with the particle-particle contact detection, while the main computational cost is shifted to the global search stage.

Approach 3. Using multi-level bounding boxes to represent particles. Each particle and its surface triangles may be decomposed into a multi-level sub-domain hierarchy using, for instance, an octree representation [35]. Each sub-domain is a bounding box of a group of triangles. Then the whole triangular mesh of a particle is represented by different sized bounding boxes depending on the level of resolution required. For a given resolution level, non-empty sub-domains at the required level will be involved in the global search. Then in the particle-particle contact stage, only triangles belonging to the sub-domains that are in the potential contact list will be checked for their intersections.

The overall performance of the first two approaches may be similar but will depend on the implementation. The third approach attempts to balance the costs occurred in the two stages depending on the resolution level specified. If only one level is used, this approach becomes the first one, or the second one if a high level of resolution is specified. Thus a proper selection of the representation level, depending on the actual surface meshes of particles, may be an important factor to achieve an optimal overall performance when applying the proposed contact models to particles that are discretised by a large number of surface triangles.

\subsection{Parallelisation}

Both $\mathbf{S}_{n}$ and $\mathbf{G}_{n}$ exhibit an additive nature as explained in detail in [29]. Due to this property, their computations can be conducted in a segment by segment fashion and in any order. Thus, the computational procedures outlined above, including the local contact search and the triangle-triangle intersection check and computation, can be fully parallelised using multiple CPU, GPU or GPGPU computing. As only a minimal level of communication overhead is incurred, a high parallel efficiency is expected. More elaboration about the parallel computing issue for the proposed contact model can be found in [29].

\subsection{Other Issues}

The presented contact volume based normal linear contact model is based on a particular form of the contact energy form. As the contact normal force is proportional to $\mathbf{S}_{n}$ or $S_{n}$, it differs from traditional overlap based contact models. Consequently, additional theoretical issues arise, such as how to determinate the critical time step and how to incorporate the contact model properly with a friction model and the standard (linear) viscous damping model to multiple contact cases. These issues are briefly addressed below. Further in-depth 
investigations will be reported later.

\section{Critical time step}

The time step affects the stability and accuracy of a DEM simulation, and is inversely proportional the total simulation time. Thus it is important from the practical point of view that a proper time step can be chosen for a given simulation. The time step $\Delta t$ is generally given by

$$
\Delta t=\lambda \Delta t_{c r}
$$

where $\lambda$ is the time step factor and $\Delta t_{c r}$ is the critical time step. A typical value for $\lambda$ is taken around $0.1 \sim 0.2$. Using a standard linear contact model with the stiffness $k_{n}$ for a contact with the equivalent mass $m, \Delta t_{c r}$ can be expressed as

$$
\Delta t_{c r}=2 \sqrt{m / k_{n}}
$$

Because the current contact model is different from the standard linear contact model, the above formula cannot be directly adopted. However, when applying the current model to a pair of equal sized spheres, it is found [29] that the normal contact force $F_{n}$ can be approximately expressed as a linear function of the overlap $\delta$ as

$$
F_{n}=\pi R k_{n} \delta
$$

where $R$ is the radius of the spheres. This equivalence of the current contact volume based linear model with the standard overlap based linear model leads to the following the critical time step expression of the current model for general contact cases

$$
\Delta t_{c r}=2 \sqrt{m / \pi R_{c} k_{n}}
$$

where $R_{c}$ is assumed to be the contact radius. For simplicity, $R_{c}$ is taken as the effective equivalent radius of the two particles concerned:

$$
R_{c}=1 /\left(1 / R_{1}+1 / R_{2}\right)
$$

where $R_{1}$ and $R_{2}$ are the equivalent radii of the two particles respectively.

Multiple contacts. A challenging issue for conventional contact models is how to deal with a multiple contact scenario, as this situation will be encountered for concave particles. A general discussion on the topic can be found in [28], while the related computational aspects for friction and damping are provided here.

As mentioned in 2.4, the current contact model can automatically distinguish if a given contact is a single or multiple contact and can correctly compute the corresponding contact geometric and normal contact force features for each individual contact in a unified manner. When friction needs to be considered, the tangential contact model is applied to each individual contact without any change from the standard treatment in DEM. However, when the damping effect needs to be considered, the damping treatment for multiple contacts is different from that of the friction. Instead of applying a viscous damping term to each contact, it is weighted by the ratio of the $S_{n}$ for this contact to the total $S_{n}$ summed up from all the contacts.

\section{Numerical Illustrations}

Several numerical examples will be presented in this section to either demonstrate the energyconserving nature of the proposed contact model for pair-wise elastic impacts between two 
concave particles, or to showcase the robustness and applicability of the proposed contact model for wider and more complex applications. Thus no comparison with any other contact model or numerical procedure will be made. This is a separated issue and will be addressed in another work to follow.

The classic central difference time integration scheme is adopted to solve the translational motion of particles. As all the shapes considered are irregular and concave, the rotational motion is solved by a symplectic time integration scheme [36] in conjunction with the use of the quaternion representation for shape orientation. The time step used in each case is automatically selected following the empirical formula (26) such that both numerical stability and reasonable accuracy can be achieved. The classic Coulomb friction model will be applied when fiction is considered. A linear viscous damping will also be added to both normal and tangential contacts when the damping effect needs to be included. Both effects are treated following the procedure outlined in Section 3.7. The critical time step is estimated used the formula (29), while the time step factor is taken to be 0.1 for the two examples with elastic impact to achieve a high accuracy, and 0.2 for the further illustrated examples.

\subsection{Energy Conservation for Elastic Impact}

In addition to a set of numerical examples that are used in [29] to verify the energy-conserving nature of the proposed contact model, two more cases will be presented in this subsection to illustrate this property. Each case consists of two concave particles that are surface triangulated. The two particles are initially separated but will be bought into contact due to the specified initial velocities. After bounce back, they will become separated again. Friction and damping effects will not be included and gravity will be de-activated. The total kinetic energy of the two-particle system, including both translational and rotational energy, will be recorded for the whole duration of the impact. The energy-conserving property can be demonstrated if the total kinetic energy remains the same before and after the impact. To facilitate this comparison, both the kinetic energy and the impact time are normalised as this will not alter the conclusion.

Case 1: Elastic impact of a banana and a pear. The banana and the pear used are the scaled versions of a sample banana and a sample pear model respectively, where the banana consists of 152 vertices and 300 triangles, and the pear has 850 vertices and 1696 triangles. Note that the realistic shape of the sharp end of the banana has been fairly well captured by the surface mesh.

Initially, the banana and the pear are randomly orientated, and are given unit but opposite

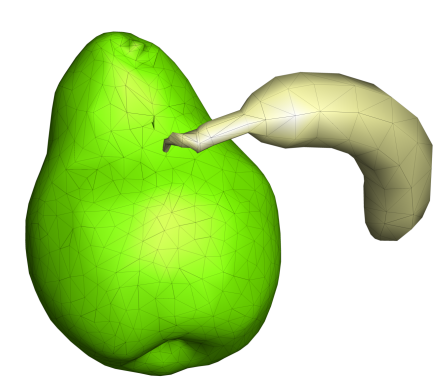

(a)

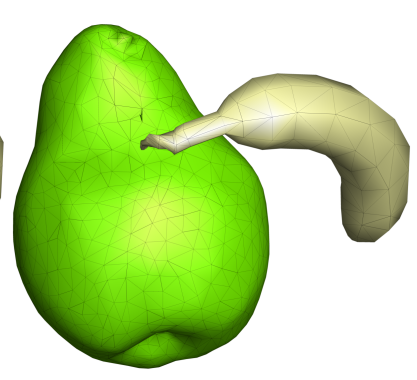

(b)

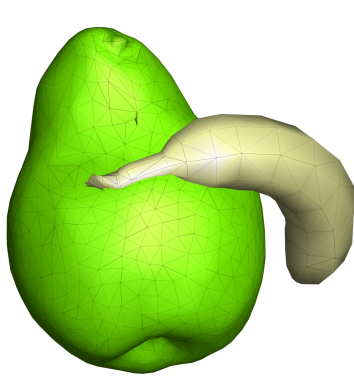

(c)

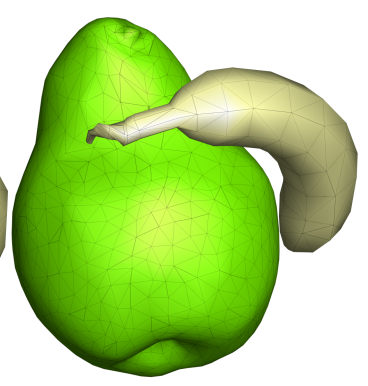

(d)

Figure 4: Elastic impact of a banana and a pear: (a) before impact; (b) at the very early contact stage; (c) at the maximum contact; and (d) after impact 


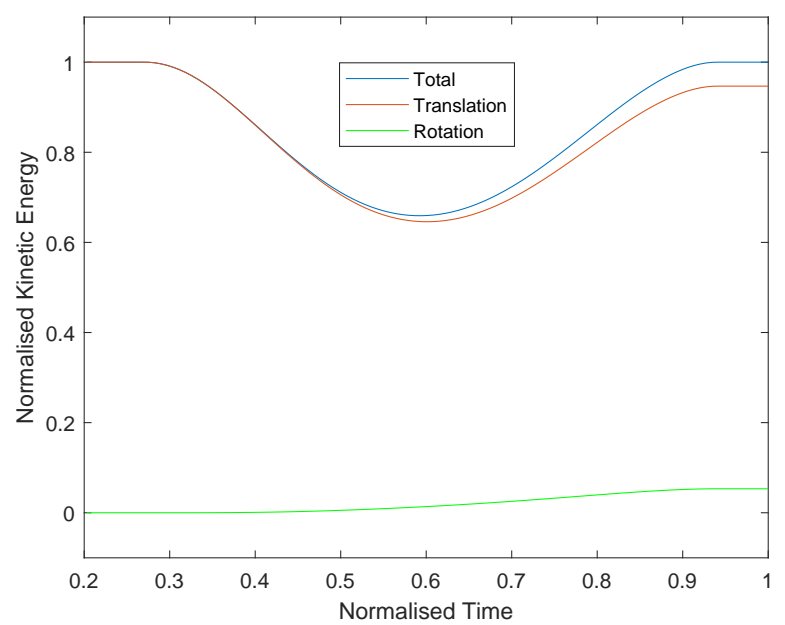

Figure 5: Elastic impact of two coffee beans: evolution of translational, rotational and total kinetic energy

initial velocities in the vertical direction, as shown in Figure 4(a). In this initial setting, the sharp end of the banana makes the contact with the pear first as shown in Figure 4(b). The penetration of this end into the pear becomes deeper as the impact progresses, and also a larger proportion of the banana makes the contact until the maximum contact reaches as shown in Figure 4(c). The configuration of the banana and the pear after the impact is displayed in Figure $4(\mathrm{~d})$.

Figure 5 shows the evolution of the (normalised) translational, rotational and total kinetic energy of the system during the impact. Because of the irregularity of the banana and the pear, a small rotational motion has developed after the impact. It is evident that the total kinetic energy is conserved in this case.

Case 2: Elastic impact of two coffee beans. The two coffee beans are obtained by scaling a scanned coffee bean model with slightly different scaling factors. The surface triangular mesh of each bean has 1095 vertices and 2186 triangles. A small value of the contact stiffness $k_{n}$ is used on purpose in order to induce a large contact penetration to test the proposed contact model for more complicated contact situations. Another reason of using a small stiffness is to prolong the impact duration so that the rotational motion of the particles can be fully developed.

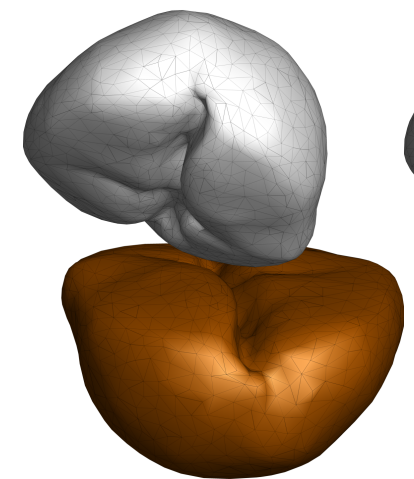

(a)

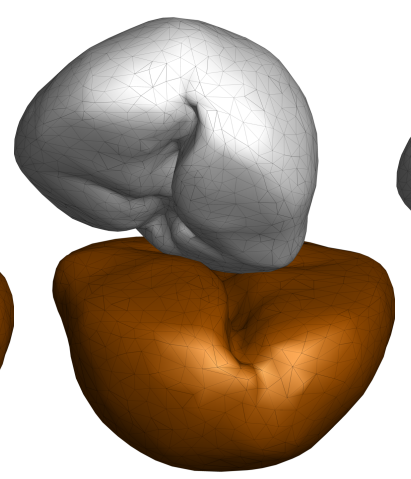

(b)

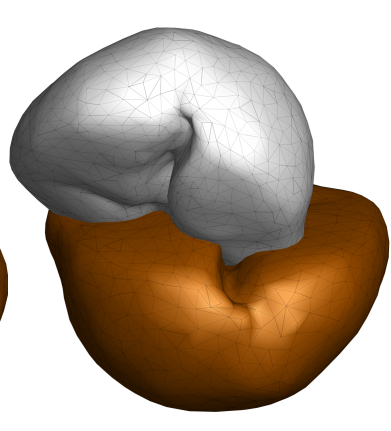

(c)

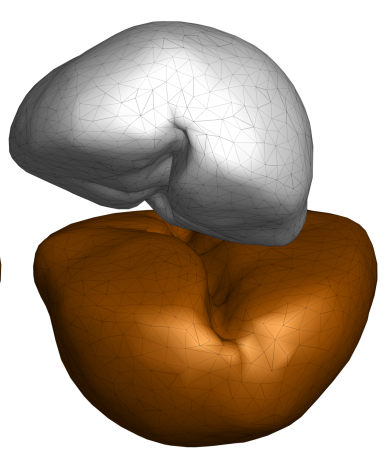

(d)

Figure 6: Elastic impact of two coffee beans: (a) before impact; (b) at the increasing penetration stage; (c) at the maximum penetration; and (d) after impact 


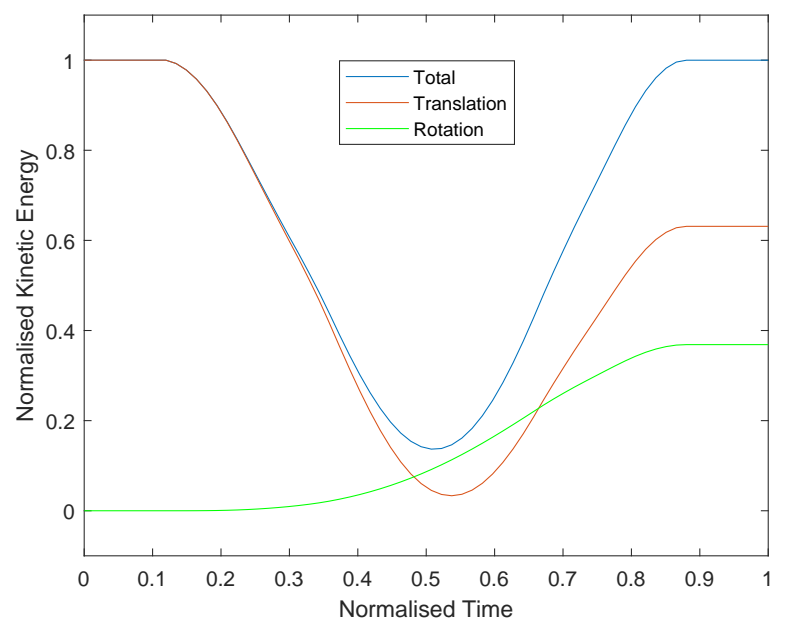

Figure 7: Elastic impact of two coffee beans: evolution of translational, rotational and total kinetic energy

Initially, the two bean are randomly orientated and are subject to an elastic collision with unit but opposite initial velocities in the vertical direction, as shown in Figure6(a). The snapshots of the impact at an instance in the first stage of impact and at the maximum penetration are displayed in Figures 6(b) and (c) respectively. The configuration of the beans after the impact is shown in Figure 6(d).

At the early stage of the impact (b), there are multiple separate contact regions but they emerge into one when the impact progresses towards the maximum penetration as shown in (c). Then in the bounce back stage, multiple and separate contacts re-appear.

Figure 7 shows the evolution of the (normalised) translational, rotational and total kinetic energy of the system during the impact. Because of the irregularity of the beans and the prolonged impact duration, a relative large rotational motion has developed after the impact. It is evident that the total kinetic energy is again conserved, even under the conditions: 1) an excessive penetration encountered; 2) both single and multiple contacts presented; and 3) rotational motion developed.

\subsection{Further Illustrative Examples}

Note that although some pair-wise contacts have been verified in the previous two cases and elsewhere, the challenge faced in a more realistic simulation is to deal with far more contact scenarios than what can be verified individually. Thus, to achieve a stable numerical simulation for a complex problem, the numerical procedures involved must be very robust.

Three more examples are presented below to illustrate the robustness and applicability of the proposed contact model and the associated numerical procedures in more realistic and complex settings. In all the examples, a coefficient of friction of 0.5 is used. The standard linear viscous damping model is extended and adopted with the current contact model. The damping ratio equivalent to a restitution of 0.1 is applied to both normal and tangential contacts. Gravity is active.

The contact detection between particles is performed using the second approach outlined in Section 3.5, where every surface triangle of a particle is represented by an axial aligned bounding box and is considered in the global search.

The first two examples involve the random deposition of concave shaped particles, including 


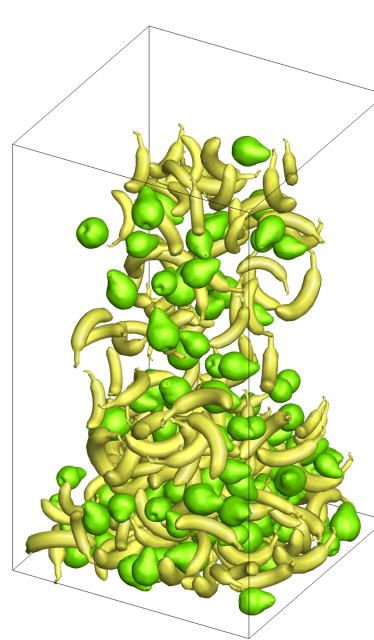

(a)

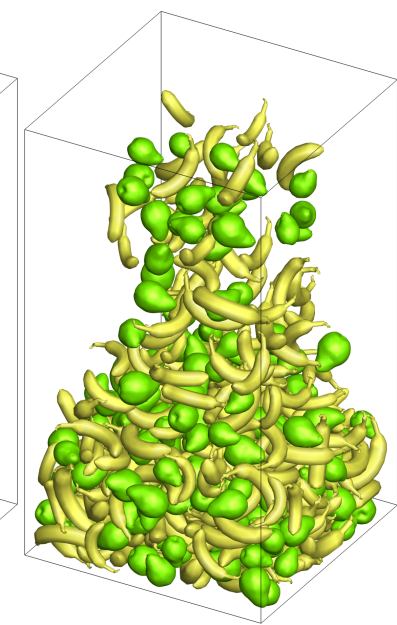

(b)

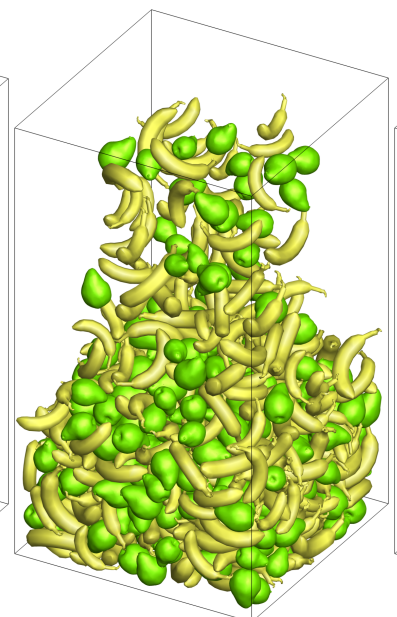

(c)

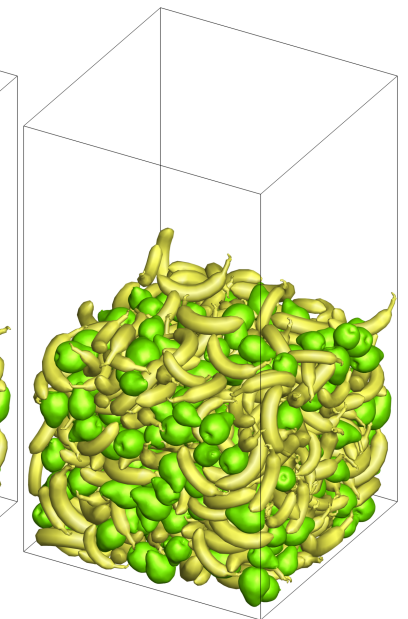

(d)

Figure 8: Random deposition of bananas and pears in a box with their packing configurations at several time instances (a) - (d)

bananas, pears and coffee beans within a box. In example 3, concave rock particles and (convex) cylindrical bars are mixed in a ball-milling setting where the boundary is rotating.

Example 1: Random packing of bananas and pears. In this example, bananas and pears are scaled versions of the triangulated banana and pear models used in case 1 above. A total number of 702 bananas and pears with slightly different sizes are randomly generated and dropped under gravity into the box. In total there are 313,307 vertices and 625,212 triangles in the system after all the particles are generated. The packing configurations of the system at four time instants are illustrated in Figure 8.

Example 2: Random packing of coffee beans. Coffee bean, based on the same triangulated model used in case 2 above are adopted in this example. A total number of 1200 coffee beans with slightly different sizes are randomly generated and dropped under gravity into the box, resulting in 1,314,000 vertices and 2,623,200 triangles in total after all the bean particles

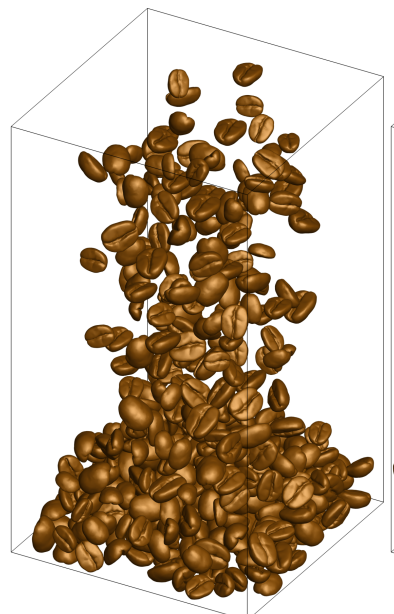

(a)

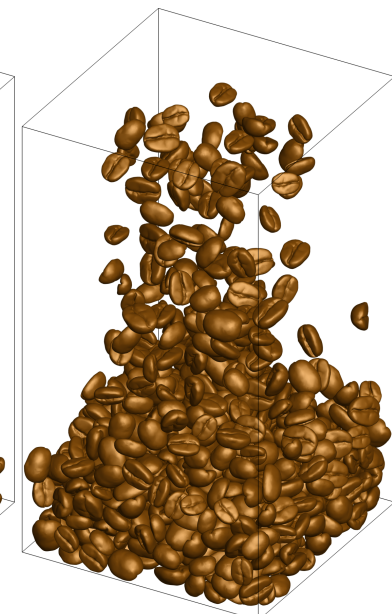

(b)

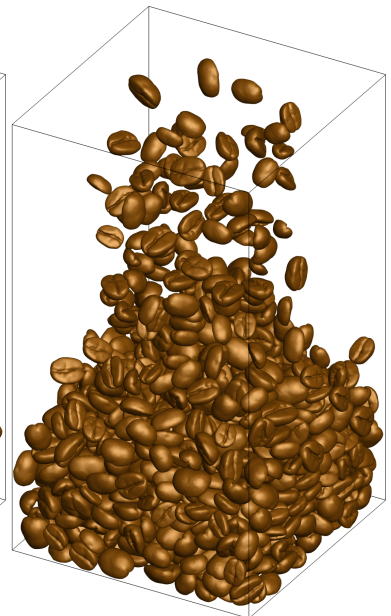

(c)

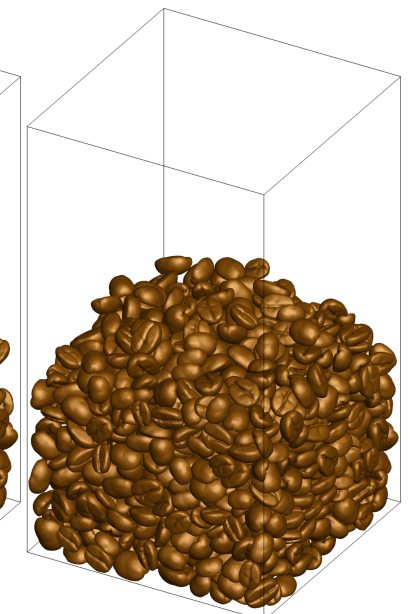

(d)

Figure 9: Random deposition of coffee beans in a box with their configurations at several time instances (a) - (d) 


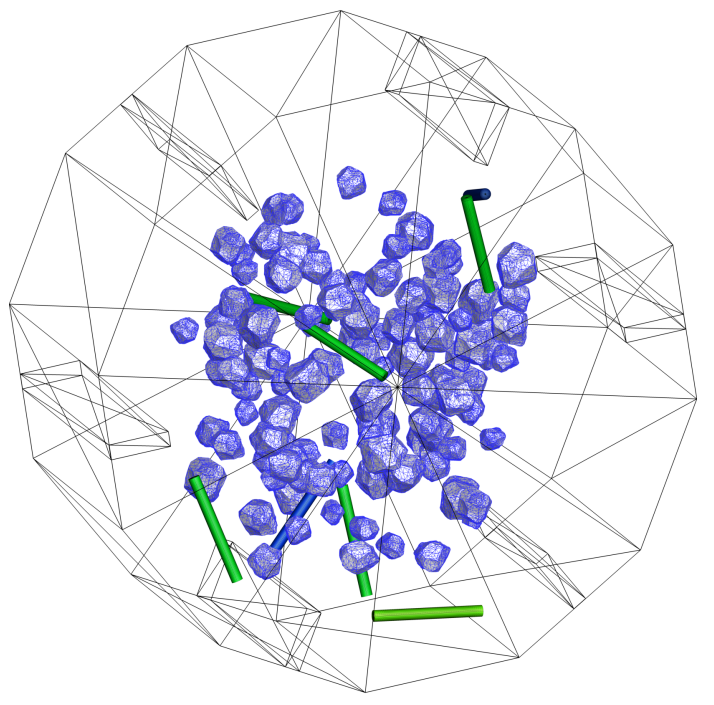

(a)

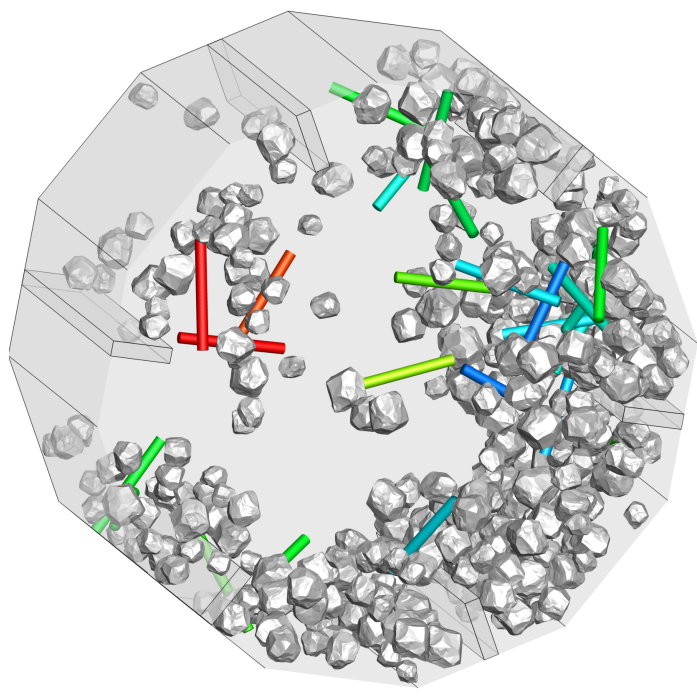

(c)

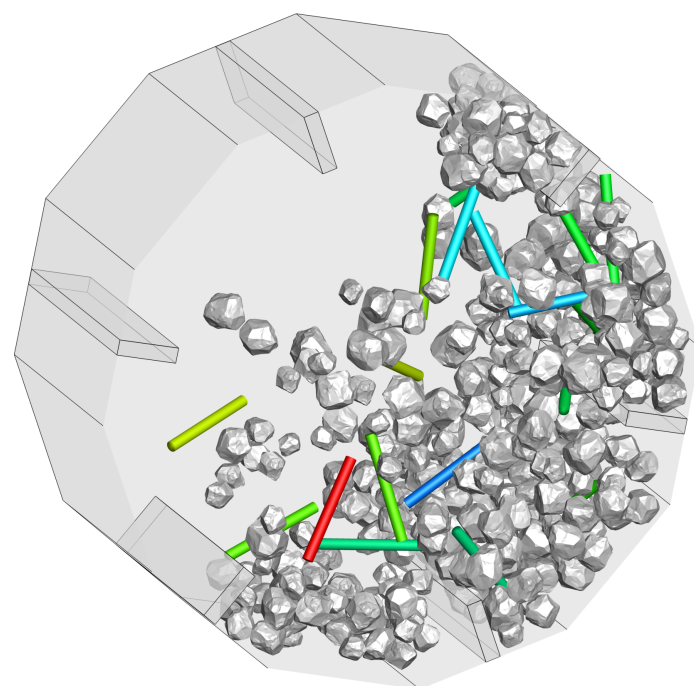

(b)

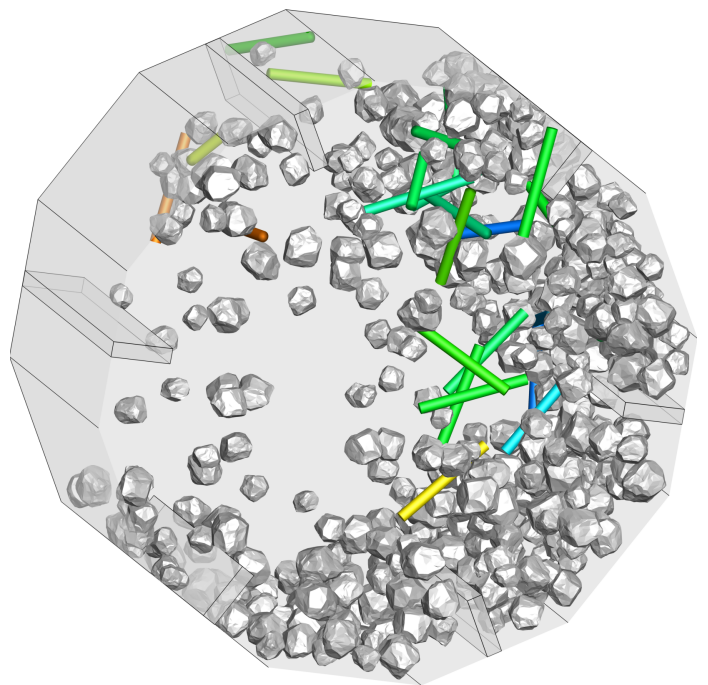

(d)

Figure 10: Mixing of rocks and metal bars in a rotating drum with baffles: triangulations of rocks and rods and the drum (a); and configurations at several time instances $t=1.5 \mathrm{~s}(\mathrm{~b})$, $3.5 \mathrm{~s}(\mathrm{c})$ and $5.5 \mathrm{~s}(\mathrm{~d})$. Colours in (b) - (d) representing velocity magnitudes of bars

are injected. The configurations of the beans at four different time instants are illustrated in Figure 9 .

In the above two examples, although various contact scenarios are encountered, a stable packing is generated at the end of each simulation. No numerical instability, such as velocity spike, has been observed, indicating that the contact model and the related numerical procedures used are stable at least for these two examples.

Example 3: Mixing of rock particles and metal bars in a rotating drum with baffles. This example attempts to simulate the operation of a ball mill in a much simplified way. A 12-sided regular rotating drum of 2 metres in diameter with six baffles attached rotates at $20 \mathrm{rpm}$ anti-clockwise. Its whole boundary including the two end covers is also represented by a surface triangular mesh. Rock particles are obtained by applying different 
scales to a concave rock sample that has 625 vertices and 1246 triangles. The equally sized cylindrical metal bars each are also discretised into a triangular mesh with 74 vertices and 144 triangles.

Both rock particles and cylindrical bars are randomly and continuously injected into the drum from a small cuboid region around the rotating axis of the drum in the early stage of the operation. 447 rock particles and 25 metal bars in total are injected into the drum which gives the total number of 281,225 vertices and 559,668 triangles for all the particles in the system.

The meshes of the drum, rock particles and metal bars at an early stage are shown in Figure 10(a). The configurations of the system at three time instances $t=1.5,3.5$, and $5.5 \mathrm{~s}$ are illustrated in Figures 10 (b)-(d) respectively. The velocity magnitudes of the metal bars are indicated by colours in the figures (where blue and red denote the lowest and highest velocities respectively).

Again, no numerical instability has been observed in this example.

\section{Concluding Remarks}

An effective generic linear normal contact model for concave particles represented by surface triangular meshes has been presented in this paper. The proposed contact model gives a systematic way to determine all the contact features involved to ensure that energy conservation will be achieved in any elastic impact between two arbitrarily shaped particles. The newly developed numerical procedures significantly reduce the computational costs involved in the model. Additional computational issues associated with the contact treatment of two surface triangulated shapes have also been discussed. The first two examples have further verified the energy-conserving property of the model for pair-wise elastic impacts between concave shaped particles. Three more complex examples have also been conducted to demonstrate the overall robustness and universal applicability of the proposed contact model and the related numerical procedures.

As the number of triangles used to represent a realistic 3D particle surface is typically very large, the total computer CPU cost required to solve a moderate sized problem can be high. Thus, using parallel computing such as GPU or GPGPU may be an ideal solution as have also been demonstrated elsewhere. Although this aspect is not exploited in the current work, the additive property of the contact geometric features in the contact model makes the corresponding computational procedures fully parallelisable, as has been discussed in Section 3, and a very high parallel speed-up can be expected. Additional issues including the estimation of a critical time step and friction and damping treatment for multiple contacts have also been discussed.

A remaining issue is how accurate that the proposed normal contact model can capture the real physical contact behaviour of two arbitrary particles. This is a challenging issue but is applied to any contact model used for almost all non-spherical particles, as no theoretical contact model similar to the Hertz model exists in these cases. As having been briefly discussed in Section 3.7, the current linear contact model for spheres is equivalent to the standard overlap based linear spring model. Thus it is expected that the current linear model should behave similarly to the standard linear spring model which is the most commonly used contact force model for non-spherical particles. However, further work is needed to adequately address the issue. 


\section{Acknowledgement}

This work is partially supported by Open Fund of State Key Laboratory of Coal Resources and Safe Mining, China University of Mining and Technology, Beijing, China [Grant No. SKLCRSM19KFA12]. This support is gratefully acknowledged.

\section{References}

[1] P. A. Cundall, and O. D. L. Strack. A discrete numerical model for granular assemblies. Geotechnique, 29(1):47-65, 1979.

[2] J.M. Ting. A robust algorithm for ellipse-based discrete element modelling of granular materials. Comput. Geotech. 13(3):175-186, 1992.

[3] T.-T. Ng. Numerical simulations of granular soil using elliptical particles. Comput. Geotech. 16(2):153-169, 1994.

[4] X. Lin, T.-T. Ng. Contact detection algorithms for three-dimensional ellipsoids in discrete element modelling. Int. J. Numer. Anal. Methods Geomech. 19(9):653-659, 1995.

[5] J. R. Williams, A. Pentland. Superquadrics and model dynamics for discrete elements in interactive design. Engineering Computations. 9:115-128, 1992.

[6] C. Wellmann, C. Lillie, and P. Wriggers. A contact detection algorithm for superellipsoids based on the common-normal concept. Engineering Computations 25(5):432-442, 2008.

[7] J. Peters, M. Hopkins, R. Kala, R. Wahl. A poly-ellipsoid particle for non-spherical discrete element method. Engineering Computations. 26(6):645-657, 2009.

[8] B. Zhang, R. Regueiro, A. Druckrey, K. Alshibli. Construction of poly-ellipsoidal grain shapes from SMT imaging on sand, and the development of a new DEM contact detection algorithm. Engineering Computations. 35:733-771, 2018.

[9] S. Zhao, J. Zhao. A poly-superellipsoid-based approach on particle morphology for DEM modeling of granular media. Int. J. Numer. Anal. Meth. Geomech., 43:2147-2169, 2019.

[10] Y. T. Feng, K. Han, D. R. J. Owen. A generic contact detection framework for cylindrical particles in discrete element modelling. Comput. Methods Appl. Mech. Engrg. 315:632$651,2017$.

[11] Y. T. Feng, D. R. J. Owen. A 2D polygon/polygon contact model: algorithmic aspects. Engineering Computations, 21:265-277, 2004.

[12] P. A. Cundall. Formulation of a three-dimensional distinct element model - Part I. A scheme to detect and represent contacts in a system composed of many polyhedral blocks. Int. J. Rock Mech. Min. Sci. \& Geomech., 25:107-116, 1988.

[13] Y. T. Feng, K. Han and D. R. J. Owen. An energy based polyhedron-to-polyhedron contact model. Proceeding of 3rd M.I.T. Conference of Computational Fluid and Solid Mechanics, pp210-214, MIT, USA, 14-17 June, 2005.

[14] Y. T. Feng, K. Han, D. R. J. Owen. Energy-conserving contact interaction models for arbitrarily shaped discrete elements. Comput. Methods Appl. Mech. Engrg. 205-208: 169-177, 2012. 
[15] W. Zhou, G. Ma, X. L. Chang, Y. Duan. Discrete modelling of rockfill materials considering the irregular shaped particles and their crushability. Engineering Computations, 32(4):1104-1120, 2015.

[16] M. A. Hopkins. Discrete element modeling with dilated particles. Engineering Computations, 21(2/3/4):422-430, 2004.

[17] L. Liu, S. Ji. Ice load on floating structure simulated with dilated polyhedral discrete element method in broken ice field. Appl. Ocean. Res. 75:5365, 2018.

[18] L. Liu, S. Y. Ji. A fast detection algorithm based on the envelope function of dilated polyhedron (in Chinese). Sci. Sin-Phys. Mech. Astron., 49:064601, 2019.

[19] L. Liu, S. Y. Ji. Bond and fracture model in dilated polyhedral DEM and its application to simulate breakage of brittle materials. Granular Matter, 21, Article No. 41, 2019. doi:10.1007/s10035-019-0896-4

[20] Z. Lai, Q. Chen, L. Huang. Fourier series-based discrete element method for computational mechanics of irregular-shaped particles. Comput. Methods Appl. Mech. Engrg. $362: 112873,2020$.

[21] J. Andrade, K. Lim, C. Avila, I. Vlahinic. Granular element method for computational particle mechanics. Comput. Methods Appl. Mech. Engrg. 241:262-274, 2012.

[22] E. J. Garboczi, J. W. Bullard. 3D analytical mathematical models of random star-shape particles via a combination of X-ray computed microtomography and spherical harmonic analysis. Advanced Powder Technology, 28(2):325-339, 2017.

[23] Z. Zhu, H. Chen, W. Xu, L Liu. Parking simulation of three-dimensional multi-sized star-shaped particles. Modelling and Simulation in Materials Science and Engineering, 22(3), article id. 035008, 2014.

[24] W. Gao, J. Wang, S. Yin, Y. T. Feng. A coupled 3D isogeometric and discrete element approach for modeling interactions between structures and granular matters. Comput. Methods Appl. Mech. Engrg. 354:441-463, 2019.

[25] W. Gao, Y. T. Feng. A coupled 3D discrete elements/isogeometric method for particle/structure interaction problems. Comp. Part. Mech., 2019. https://doi.org/10.1007/s40571-019-00267-8.

[26] S. Ji. and L. Lu. Computational Granular Mechanics and Its Engineering Applications. Springer, 2020. ISBN 978-981-15-3304-4.

[27] Y. T. Feng. A general contact theory for non-spherical particles. In: X. Li, Y. Feng, G. Mustoe (eds), Proceedings of 7th International Conference on Discrete Element Methods (Dem 2016). Springer, pp 29-35, 2017.

[28] Y. T. Feng. An energy-conserving contact theory for discrete element modelling of arbitrarily shaped particles: Basic framework and general contact model. Comput. Methods Appl. Mech. Engrg. 373:113454, 2021

[29] Y. T. Feng. An energy-conserving contact theory for discrete element modelling of arbitrarily shaped discrete elements: Contact volume based model and computational issues. Comput. Methods Appl. Mech. Engrg. 373:113493, 2021 
[30] Y. T. Feng. An effective energy-conserving contact modelling strategy for spherical harmonic particles represented by surface triangular meshes with automatic simplification. Comput. Methods Appl. Mech. Engrg. 2021 (to appear).

[31] B. Nassauer, T. Liedke and M. Kuna Polyhedral particles for the discrete element method: Geometry representation, contact detection and particle generation. Granular Matters, 15:85-94, 2013.

[32] N. Govender, N. Daniel, C.-Y. Wu, J. Khinast, P. Pizette, X. Wenjie. Hopper flow of irregularly shaped particles (non-convex polyhedra): GPU-based DEM simulation and experimental validation. Chemical Engineering Science. 188:34-51, 2018.

[33] A. H. Barr. Superquadrics and Angle-Preserving Transformations. IEEE Computer Graphics and Applications. 1(1):11-23, 1981.

[34] D. R. Owen, Y. T. Feng, E. de Souza Neto, F. Wang, M. G. Cottrel, F. A. Pires, J. Yu. The modelling of Multi-fracturing Solids and Particulate Media. International Journal for Numerical Methods in Engineering. 60(1): 317-340, 2004.

[35] D. Meagher. Octree encoding: A new technique for the representation, manipulation and display of arbitrary 3-D objects by computer. Rensselaer Polytechnic Institute, Technical Report IPL-TR-80-111, October 1980.

[36] A. Dullweber, B. Leimkuhlera and R. McLachlanb: Symplectic splitting methods for rigid body molecular dynamics. J. Chem. Phys. 107(15):5831-5851, 1997. 